Pacific Journal of Mathematics

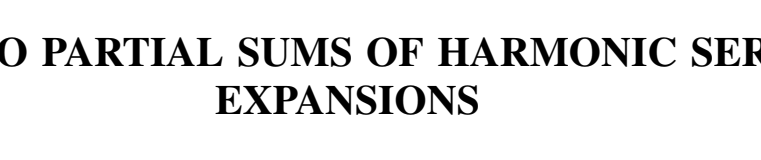




\section{CESÀRO PARTIAL SUMS OF HARMONIC SERIES EXPANSIONS}

\section{S. ROBERTSON}

1. Introduction. Let the harmonic function $v(r, \theta)$ have the sine series expansion

$$
v(r, \theta)=\sum_{1}^{\infty} a_{\nu} r^{\nu} \sin \nu \theta,
$$

convergent for $0 \leqq r<1$ and suppose that $v(r, \theta)$ is non-negative for $0<\theta<\pi$. Denote the $n$th partial sum of (1.1) by

$$
S_{n}^{(0)}(r, \theta)=\sum_{1}^{n} a_{\nu} r^{\nu} \sin \nu \theta
$$

and the $n$th Cesàro partial sum of order $k$, by

$$
S_{n}^{(k)}(r, \theta)=\sum_{1}^{n} C_{k}^{n+k-\nu} a_{\nu} r^{\nu} \sin \nu \theta, \quad k=1,2, \cdots .
$$

It was shown by Fejér [2, p. 61] and Szász [8] that when $v(r, \theta) \geqq 0$ for $0<\theta<\pi, 0<r<1$, then $S_{n}^{(0)}(r, \theta)$ is also non-negative for all $n$ when $0<\theta<\pi, 0<r \leqq 1 / 4$, and the constant $1 / 4$ is sharp. Fejér [2] showed that the functions $S_{n}^{(3)}(1, \theta)$ are also non-negative for all $n$, $0<\theta<\pi$. In addition, Szász [8] showed that there exists an $R_{n}^{(0)}$, depending upon $n$ only, so that $S_{n}^{(0)}(r, \theta) \geqq 0$ for $0<r \leqq R_{n}^{(0)}, 0 \leqq \theta \leqq \pi$, but not always for $r>R_{n}^{(0)}$, and that

$$
R_{n}^{(0)}=1-3 \frac{\log n}{n}+\frac{\log \log n}{n}+O(1 / n) .
$$

In this paper we shall extend the results of Szász to Cesàro partial sums of integral order $k, k=1,2,3$. For $k=3$ the theorem obtained is a sharpened form of the theorem of Fejér [2]. We prove the following :

THEOREM 1. Let the harmonic series expansion

$$
v(r, \theta)=\sum_{1}^{\infty} a_{\nu} r^{\nu} \sin \nu \theta
$$

be convergent for $0 \leqq r<1$ and let $v(r, \theta) \geqq 0$ for $0<\theta<\pi, 0<r<1$. Then for $k=0,1,2,3$ there exists a positive number $R_{n}^{(k)}$ depending upon $n$ only, so that

Received by the editors February 27, 1958, and in revised form June 3, 1958. The author wishes to express his appreciation to the referee for helpful suggestions. 


$$
S_{n}^{(k)}(r, \theta) \geqq 0 \quad \text { for } \quad 0 \leqq r \leqq R_{n}^{(k)}, \quad 0 \leqq \theta \leqq \pi,
$$

but not always for $r>R_{n}^{(k)}$, and that

$$
R_{n}^{(k)}=1-(3-k) \frac{\log n}{n}+\frac{\log \log n}{n}-\frac{g_{k}+o(1)}{n}, \quad k=0,1,2,
$$

where

$$
g_{k}=\log \left[\left\{1+\frac{k+1}{6}\left(1+(-1)^{k}\right)\right\} \mu\right] \text {, }
$$

and where

$$
\mu= \begin{cases}1 \text { for } n & \text { even } \\ \max _{\pi \leqq h \leqq 3 \pi / 2} & |\sin h| h \mid=0.217 \cdots \text { for } n \text { odd } ;\end{cases}
$$

and

$$
\begin{gathered}
R_{2 n}^{(3)}=1, \quad R_{2 n-1}^{(3)}>1, \quad n=1,2, \cdots, \\
\limsup _{n \rightarrow \infty}(2 n-1)\left(R_{2 n-1}^{(3)}-1\right) \leqq \alpha_{0}=1.07 \ldots
\end{gathered}
$$

where $\alpha_{0}$ is the positive root of the equation

$$
3-\alpha-3 \mu e^{\alpha}=0 .
$$

Moreover, $R_{n}^{(k)}$ is the largest $r$ for which $\psi_{n}^{(k)}(r, \theta)$ is non-negative for all $\theta$, where $\psi_{n}^{(k)}(r, \theta)$ is defined for $k=0,1,2,3$ by the equations (2.18), (2.19), (2.20), and (2.21).

Since $v(r, \theta)$ in (1.1) may be regarded as the imaginary part of the analytic function

$$
f(z)=\sum_{1}^{\infty} a_{\nu} z^{\prime}, \quad z=r e^{i \theta}, \quad r<1, a_{\nu} \text { real, }
$$

the property $v(r, \theta) \geqq 0$ for $0<\theta<\pi$ may be interpreted by saying that $f(z)$ is typically-real in the unit circle, that is $\Im f(z)>0$ for $\Im z>0$, and $\Im f(z)<0$ for $\Im z<0,|z|<1$. In this case

$$
F(z)=\int_{0}^{z} \frac{f(z)}{z} d z
$$

is schlicht and convex in the direction of the imaginary axis for $|z|<1$. For from (1.10) we have

$$
\frac{\partial}{\partial \theta} \Re F\left(r e^{i \theta}\right)=-\Im z F^{\prime}(z)=-\Im f(z)<0
$$

for $|z|<1,0<\theta<\pi$. 
Definition. Let $\mathscr{F}_{r}$ and $\mathscr{F}_{r}^{*}$ denote the families of functions

$$
F(z)=z+b_{2} z^{2}+\cdots+b_{n} z^{n}+\cdots
$$

which are regular, real on the real axis, schlicht and convex in the direction of the imaginary axis in $|z|<r$, and in $|z| \leqq r$ respectively.

With the help of Theorem 1 we then obtain the following.

\section{THEOREM 2. Let}

$$
F(z)=z+b_{2} z^{2}+\cdots+b_{n} z^{n}+\cdots
$$

be a member of the family $\mathscr{F}_{1}$. Then for $n=1,2,3, \cdots$ the $n$th Cesaro partial sum of order one of (1.13) is a member of $\mathscr{F}_{1 / 2}^{*}$. The radius $1 / 2$ cannot be replaced by a larger number. Also the nth Cesàro partial sum of order $k, k=0,1,2,3$, is a member of $\mathscr{F}_{p_{k}}^{*}$ where

$$
\begin{array}{ll}
\rho_{0}=1-3 n^{-1} \log n+n^{-1} \log \{(3 / 4-\varepsilon) \log n\}, & n>n_{0}(\varepsilon), \\
\rho_{\mathrm{L}}=1-2 n^{-1} \log n+n^{-1} \log \{(1-\varepsilon) \log n\}, & n>n_{1}(\varepsilon), \\
\left.\mu_{2}=1-n^{-1} \log n+n^{-1} \log \{1 / 2-\varepsilon) \log n\right\}, & n>n_{2}(\varepsilon), \\
\rho_{3} \begin{cases}=1, & n \text { even, } \\
>1, & n \text { odd, }\end{cases}
\end{array}
$$

and where $\varepsilon>0$ is arbitrarily small and $n_{k}(\varepsilon), k=0,1,2$, are positive integers depending only upon $\varepsilon$. The radii $\rho_{k}$ are sharp to within $O(1 / n)$.

2. Preliminary formulas. Before we proceed to the proof of Theorem 1 we shall mention several formulas which will be needed. The following sums are easily calculated:

$$
\begin{aligned}
S(z) & =z+2 z^{2}+\cdots+n z^{n}+\cdots=z(1-z)^{-2}, \\
S_{n}^{(0)}(z) & =z+2 z^{2}+\cdots+n z^{n}=\left\{z-(n+1) z^{n+1}+n z^{n+2}\right\}(1-z)^{-2} \\
S_{n}^{(k)}(z) & =S_{1}^{(k-1)}(z)+S_{2}^{(k-1)}(z)+\cdots+S_{n}^{(k-1)}(z), \quad k=1,2, \cdots \\
S_{n}^{(k)}(z) & =C_{k}^{n+k-1} z+2 C_{k}^{n+k-2} z^{2}+\cdots+n C_{k}^{k} z^{n}, \\
S_{n}^{(1)}(z) & =\left\{n z-(n+2) z^{2}+(n+2) z^{n+2}-n z^{n+3}\right\}(1-z)^{-3} \\
S_{n}^{(2)}(z) & =\frac{1}{2 !}\left\{n(n+1) z-2 n(n+3) z^{2}+(n+2)(n+3) z^{3}\right. \\
& \left.\quad-2(n+3) z^{n+3}+2 n z^{n+4}\right\}(1-z)^{-4},
\end{aligned}
$$

$$
S_{n}^{(3)}(z)=\frac{1}{3 !}\left\{n(n+1)(n+2) z-3 n(n+1)(n+4) z^{2}\right.
$$




$$
\begin{aligned}
+3 n(n+3)(n+4) z^{3}- & (n+2)(n+3)(n+4) z^{4} \\
& \left.+6(n+4) z^{n+4}-6 n z^{n+5}\right\}(1-z)^{-5},
\end{aligned}
$$

(2.8) $S_{n}^{(k)}(z)=\frac{1}{k !}\left\{\sum_{m=1}^{k+1}(-1)^{m-1} \prod_{p=0}^{k-m}(n+p) \prod_{q=k+3-m}^{k+1}(n+q) z^{m}\right.$

$$
\begin{array}{r}
\left.+(-1)^{k-1} k !(n+k+1) z^{n+k+1}+(-1)^{k} k ! n z^{n+k+2}\right\}(1-z)^{-k-2}, \\
k=0,1, \cdots,
\end{array}
$$

where $\Pi_{p=i}^{j}(n+p)$ is defined to be 1 if $i>j$.

Let

$$
f(z)=\sum_{1}^{\infty} a_{\nu} z^{\nu}, \quad \quad a_{1}>0, a_{\nu} \text { real, }
$$

be regular and typically-real in $|z|<1$, which is to say that $v(r, \theta)=$ $\Im f\left(r e^{i \theta}\right)$ is non-negative for $0 \leqq \theta \leqq \pi, 0<r<1$, and $f(z)$ is real on the real axis. As I have shown elsewhere [3] the function $f(z)$ may be represented by the Stieltjes integral

$$
f(z)=\frac{a_{1}}{\pi} \int_{0}^{\pi} P(z, \phi) d \alpha(\phi),
$$

where $\alpha(\phi)$ is a non-decreasing real function of $\phi$ in the interval $[0, \pi]$, and where $P(z, \phi)$ is the typically-real, schlicht and star-like function

$$
P(z, \phi) \equiv z\left(1-2 z \cos \phi+z^{2}\right)^{-1}=\sum_{1}^{\infty} \frac{\sin \nu \phi}{\sin \phi} z^{\nu} .
$$

For $\phi=0$ we have $P(z, 0)=S(z)$ where $S(z)$ is defined as in (2.1). From (2.10) we have immediately that

$$
S_{n}^{(k)}(r, \theta)=\frac{a_{1}}{\pi} \int_{0}^{\pi} \Im P_{n}^{(k)}\left(r e^{i \theta}, \phi\right) d \alpha(\phi)
$$

where $P_{n}^{(k)}\left(r e^{i \theta}, \phi\right)$ is the $n$th Cesàro partial sum of order $k$ of the power series for $P(z, \phi)$ given in (2.11),

$$
\Im P_{n}^{(k)}\left(r e^{i \theta}, \phi\right)=\sum_{\nu=1}^{n} C_{k}^{n+k-\nu} \gamma^{\nu} \frac{\sin \nu \phi}{\sin \phi} \sin \nu \theta .
$$

By a lemma of L. Fejér [8], [9], [4], it follows that

$$
\Im P_{n}^{(k)}\left(r e^{i \theta}, \phi\right) \geqq 0, \quad 0 \leqq \phi \leqq \pi, 0 \leqq \theta \leqq \pi,
$$

if, and only if,

$$
\Im S_{n}^{(k)}\left(r e^{i \theta}\right)=\Im P_{n}^{(k)}\left(r e^{i \theta}, 0\right)=\sum_{\nu=1}^{n} \nu C_{k}^{n+k-\nu} r^{\nu} \sin \nu \theta \geqq 0, \quad 0 \leqq \theta \leqq \pi .
$$

Thus, the behavior of the Cesàro partial sums of the Koebe function 
$z(1-z)^{-2}$ determines the extremes to which the Cesàro partial sums of the series expansion of an arbitrary typically-real function $f(z)$ exhibit their properties. Therefore, in order to prove Theorem 1 for the imaginary part of an arbitrary typically-real function $f(z)$ we may confine ourselves to proving these results merely for the Koebe function $S(z)=z(1-z)^{-2}$. For this function the partial sums

$$
S_{n}^{(0)}(z)=\left\{z-(n+1) z^{n+1}+n z^{n+2}\right\}(1-z)^{-2}
$$

are known to be schlicht and star-like with respect to the origin in [1]

$$
|z| \leqq 1-3 n^{-1} \log n, \quad n>n_{0},
$$

and $\grave{a}$ fortiori typically-real in the same radius.

From formulas (2.2), (2.5), (2.6), (2.7), on letting $z=r e^{i \theta}$ we obtain by simple, straightforward but long computations the following additional formulas which we shall need.

$$
k !|1-z|^{2 k+4} \Im S_{n}^{(k)}\left(r e^{i \theta}\right)=r \sin \theta \psi_{n}^{(k)}(r, \theta), \quad k=0,1,2,3,
$$

where

$$
\begin{aligned}
& \psi_{n}^{(0)}(r, \theta)=1-r^{2}-(n+1) r^{n+2} \frac{\sin (n-1) \theta}{\sin \theta} \\
& +r^{n+1}\left(2 n+2+n r^{2}\right) \frac{\sin n \theta}{\sin \theta}-r^{n}\left(n+1+2 n r^{2}\right) \frac{\sin (n+1) \theta}{\sin \theta} \\
& +n r^{n+1} \sin (n+2) \theta \\
& \sin \theta
\end{aligned}
$$

$$
\begin{aligned}
\psi_{n}^{(1)}(r, \theta) & =\left\{n+6 r^{2}-(n+2) r^{4}\right\}-\left\{(n+2) r-n r^{3}\right\} \frac{\sin 2 \theta}{\sin \theta} \\
- & (n+2) r^{n+4} \frac{\sin (n-1) \theta}{\sin \theta}+\left\{(3 n+6) r^{n+3}+n r^{n+5}\right\} \frac{\sin n \theta}{\sin \theta} \\
- & \left\{(3 n+6) r^{n+2}+3 n r^{n+4}\right\} \frac{\sin (n+1) \theta}{\sin \theta} \\
& +\left\{(n+2) r^{n+1}+3 n r^{n+3}\right\} \frac{\sin (n+2) \theta}{\sin \theta}-n r^{n+2} \frac{\sin (n+3) \theta}{\sin \theta}
\end{aligned}
$$

(2.20) $\psi_{n}^{(2)}(r, \theta)=\left\{n(n+1)+\left(2 n^{2}+18 n\right) r^{2}\right.$

$$
\begin{aligned}
& \left.-\left(2 n^{2}-6 n-36\right) r^{4}-\left(n^{2}+5 n+6\right) r^{6}\right\} \\
& -r\left\{2 n^{2}+6 n+(16 n+24) r^{2}-\left(2 n^{2}+6 n\right) r^{4}\right\} \frac{\sin 2 \theta}{\sin \theta} \\
& +r^{2}\left\{n^{2}+5 n+6-\left(n^{2}+n\right) r^{2}\right\} \frac{\sin 3 \theta}{\sin \theta} \\
& -(2 n+6) r^{n+6} \frac{\sin (n-1) \theta}{\sin \theta}+\left(8 n+24+2 n r^{2}\right) r^{n+5} \frac{\sin n \theta}{\sin \theta}
\end{aligned}
$$




$$
\begin{gathered}
-\left(12 n+36+8 n r^{2}\right) r^{n+4} \sin (n+1) \theta \\
\sin \theta \\
+\left(8 n+24+12 n r^{2}\right) r^{n+3} \sin (n+2) \theta \\
\sin \theta \\
-\left(2 n+6+8 n r^{2}\right) r^{n+2} \sin (n+3) \theta+2 n r^{n+3} \sin (n+4) \theta, \\
\sin \theta
\end{gathered}
$$

$(2.21) \quad \psi_{n}^{(3)}(r, \theta)=\left\{n(n+1)(n+2)+5 n(n+1)(n+8) r^{2}+60 n(n+4) r^{4}\right.$

$$
\left.-5(n+3)\left(n^{2}-16\right) r^{6}-(n+2)(n+3)(n+4) r^{8}\right\}
$$$$
-\left\{3 n(n+1)(n+4) r+5\left(n^{3}+15 n^{2}+32 n\right) r^{3}\right.
$$$$
\left.-5(n+4)\left(n^{2}-7 n-12\right) r^{5}-3 n(n+3)(n+4) r^{7}\right\} \frac{\sin 2 \theta}{\sin \theta}
$$$$
+\left\{3 n(n+3)(n+4) r^{2}+30(n+2)^{2} r^{4}\right.
$$

$$
\left.-3 n(n+1)(n+4) r^{6}\right\} \frac{\sin 3 \theta}{\sin \theta}
$$$$
-\left\{(n+2)(n+3)(n+4) r^{3}-n(n+1)(n+2) r^{5}\right\} \frac{\sin 4 \theta}{\sin \theta}
$$$$
-(6 n+24) r^{n+8} \frac{\sin (n-1) \theta}{\sin \theta}
$$

$$
\begin{gathered}
+\left\{(30 n+120) r^{n+7}+6 n r^{n+9}\right\} \frac{\sin n \theta}{\sin \theta} \\
-\left\{(60 n+240) r^{n+6}+30 n r^{n+8}\right\} \begin{array}{c}
\sin (n+1) \theta \\
\sin \theta
\end{array} \\
+\left\{(60 n+240) r^{n+5}+60 n r^{n+7}\right\} \frac{\sin (n+2) \theta}{\sin \theta} \\
-\left\{(30 n+120) r^{n+4}+60 n r^{n+6}\right\} \frac{\sin (n+3) \theta}{\sin \theta} \\
+\left\{(6 n+24) r^{n+3}+30 n r^{n+5}\right\} \frac{\sin (n+4) \theta}{\sin \theta} \\
\quad-6 n r^{n+4} \frac{\sin (n+5) \theta}{\sin \theta} .
\end{gathered}
$$

3. Proof of Theorem 1 for $k=1$. We proceed now to the proof of Theorem 1. For $k=0$ Theorem 1 follows from the theorem of Szász [8]. For $k=1$ we have $\Im S_{n}^{(1)}\left(r e^{i \theta}\right) \geqq 0$ for $0<\theta<\pi$ provided $\psi_{n}^{(1)}(r, \theta)$ $\geqq 0$ for all $\theta$ and $r \leqq R_{n}^{(1)}$. From (2.19) we must determine the largest $r$ for which

(3.1) $\psi_{n}^{(1)}(r, \theta)=\left\{n+6 r^{2}-(n+2) r^{4}\right\}-\left\{(n+2) r-n r^{3}\right\} \frac{\sin 2 \theta}{\sin \theta}$ 


$$
\begin{aligned}
& -(n+2) r^{n+1} \frac{\sin (n-1) \theta}{\sin \theta}+\left\{(3 n+6) r^{n+3}+n r^{n+5}\right\}-\frac{\sin n \theta}{\sin \theta} \\
& -\left\{(3 n+6) r^{n+2}+3 n r^{n+4}\right\} \frac{\sin (n+1) \theta}{\sin \theta} \\
& +\left\{(n+2) r^{n+1}+3 n r^{n+3}\right\} \frac{\sin (n+2) \theta}{\sin \theta}-n r^{n+2} \frac{\sin (n+3) \theta}{\sin \theta}
\end{aligned}
$$

is non-negative for all $\theta$. We rewrite $\psi_{n}^{(1)}(r, \theta)$ in the form

$$
\psi_{n}^{(1)}(r, \theta)=A+B(1-\cos \theta)-r^{n} \sum_{j=0}^{4}(-1)^{j} C_{j} \frac{\sin (n-1+j) \theta}{\sin \theta}
$$

where

$$
\begin{array}{ll}
A=n+6 r^{2}-(n+2) r^{4}-(2 n+4) r+2 n r^{3} \\
& =n(1-r)^{3}(1+r)-2 r(2+r)(1-r)^{2}, \\
B=(2 n+4) r-2 n r^{3}, & \\
C_{0}=(n+2) r^{4}, & C_{1}=(3 n+6) r^{3}+n r^{5}, \\
C_{2}=(3 n+6) r^{2}+3 n r^{4}, & C_{3}=(n+2) r+3 n r^{3}, \quad C_{4}=n r^{2} .
\end{array}
$$

Let

$$
\begin{aligned}
& r=e^{-\varepsilon}, \quad \varepsilon=\frac{2 \log n}{n}-\frac{\log \log n}{n}+\frac{p}{n}, \quad r^{n}=\frac{\log n}{n^{2}} e^{-p}, \\
& 1-r=1-2 \frac{\log n}{n^{2}}+\frac{\log \log n}{n}-\frac{p}{n}+O\left(\left(\frac{\log n}{n}\right)^{2}\right) .
\end{aligned}
$$

Then

$$
A \cong 16 \frac{(\log n)^{3}}{n^{2}}, \quad B \cong 8 \log n
$$

For fixed $k$ we have

$$
r^{k}=e^{-k \varepsilon}=1-k \varepsilon+\frac{k^{2}}{2} \varepsilon^{2}+O\left(\varepsilon^{3}\right),
$$

so that

$$
\begin{aligned}
& C_{0}=(n+2)-(4 n+8) \varepsilon+8 n \varepsilon^{2}+O\left(\varepsilon^{3} n\right) \\
& C_{1}=(4 n+6)-(14 n+18) \varepsilon+26 n \varepsilon^{2}+O\left(\varepsilon^{3} n\right) \\
& C_{2}=(6 n+6)-(18 n+12) \varepsilon+30 n \varepsilon^{2}+O\left(\varepsilon^{3} n\right) \\
& C_{3}=(4 n+2)-(10 n+2) \varepsilon+14 n \varepsilon^{2}+O\left(\varepsilon^{3} n\right) \\
& C_{4}=n \quad-2 n \varepsilon+2 n \varepsilon^{2}+O\left(\varepsilon^{3} n\right) .
\end{aligned}
$$

To obtain an asymptotic estimate for $\psi_{n}^{(1)}(r, \theta)$ in (3.2) we shall make use of the following lemma. 
Lemma 1. Let $\alpha_{j}, j=0,1, \cdots, 5$, be constants. Let $n$ be a positive integer and let

$$
\begin{aligned}
S & =\sum_{j=0}^{5}(-1)^{j} \alpha_{j} \frac{\sin (n-1+j) \theta}{\sin \theta} \\
& =\frac{\sin (n-1) \theta}{\sin \theta} \sum_{j=0}^{5}(-1)^{j} \alpha_{j} \cos j \theta+\cos (n-1) \theta \sum_{j=1}^{5}(-1)^{j} \alpha_{j} \frac{\sin j \theta}{\sin \theta} .
\end{aligned}
$$

(a) If $\sum_{j=0}^{5}(-1)^{j} \alpha_{\jmath}=0$, then

$$
S=\{n(1-\cos \theta)+1\} \cdot \max _{j}\left|\alpha_{j}\right| \cdot O(1) \text { as } n \rightarrow \infty \text {. }
$$

(b) If in addition to (a), $\sum_{j=1}^{5}(-1)^{j} j^{2} \alpha_{j}=0$, then

$$
\dot{S}=\left\{n(1-\cos \theta)^{2}+1\right\} \cdot \max _{j}\left|\alpha_{j}\right| \cdot O(1) \text { as } n \rightarrow \infty .
$$

(c) If in addition to (a) and (b), $\sum_{j=1}^{5}(-1)^{j} j a_{j}=0$, then

$$
S=\left\{n(1-\cos \theta)^{2}+(1-\cos \theta)\right\} \cdot \max _{j}\left|\alpha_{j}\right| \cdot O(1) \text { as } n \rightarrow \infty .
$$

The lemma is easily obtained by considering the limits

$$
\lim _{\theta \rightarrow 0} \frac{\sum_{j=0}^{5}(-1)^{j} \alpha_{j} \cos j \theta}{1-\cos \theta}, \quad \lim _{\theta \rightarrow 0} \frac{\sum_{j=1}^{5}(-1)^{j} \alpha_{j} \sin j \theta}{\sin \theta-\frac{1}{2} \sin 2 \theta} .
$$

From (3.2) and (3.5) we obtain

$$
\psi_{n}^{(1)}(r, \theta)=A+B(1-\cos \theta)-r^{n}\left[D_{0}-D_{1} \varepsilon+D_{2} \varepsilon^{2}-D_{3} \varepsilon^{3}\right]
$$

where

$$
\begin{aligned}
D_{0}=(n+2) \frac{\sin (n-1) \theta}{\sin \theta}-(4 n+6) \frac{\sin n \theta}{\sin \theta}+(6 n+6) \frac{\sin (n+1) \theta}{\sin \theta} \\
=4 n \frac{\sin (n+1) \theta}{\sin \theta}(1-\cos \theta)^{2} \\
\quad-4 \frac{\sin (n-1) \theta}{\sin \theta}(1+2 \cos \theta)(1-\cos \theta)^{2} \\
\sin \theta \\
=4 \sin (n+3) \theta \\
\sin \theta \\
=4 n \frac{\sin (n+1) \theta}{\sin \theta}(1-\cos \theta)^{2}+(1-\cos \theta) O(n) .
\end{aligned}
$$


(3.8) $D_{1}=(4 n+8) \frac{\sin (n-1) \theta}{\sin \theta}-(14 n+18) \frac{\sin n \theta}{\sin \theta}$

$$
\begin{aligned}
& +(18 n+12) \frac{\sin (n+1) \theta}{\sin \theta}-(10 n+2) \frac{\sin (n+2) \theta}{\sin \theta} \\
& +2 n \frac{\sin (n+3) \theta}{\sin \theta} \\
= & \{n(1-\cos \theta)+1\} \cdot n \cdot O(1) .
\end{aligned}
$$

(3.9) $D_{2}=8 n \frac{\sin (n-1) \theta}{\sin \theta}-26 n \frac{\sin n \theta}{\sin \theta}+30 n \frac{\sin (n+1) \theta}{\sin \theta}$

$$
=\{n(1-\cos \theta)+1\} \cdot n \cdot O(1) . \quad \frac{\sin (n+2) \theta+2 n \frac{\sin (n+3) \theta}{\sin \theta}}{\sin \theta}
$$

(3.10) $D_{3}=\sum_{j=0}^{4} O(n) \frac{\sin (n-1+j) \theta}{\sin \theta}=O\left(n^{2}\right)$.

(3.11) $D_{0}-D_{1} \varepsilon+D_{2} \varepsilon^{2}-D_{3} \varepsilon^{3}=4 n \frac{\sin (n+1) \theta}{\sin \theta}(1-\cos \theta)^{2}$

$$
\begin{aligned}
& +(1-\cos \theta) O(n)-\left\{n^{2}(1-\cos \theta)+n\right\} O(1) \frac{\log n}{n} \\
& +\left\{n^{2}(1-\cos \theta)+n\right\} O(1)\left(\frac{\log n}{n}\right)^{2}+O\left(n^{2}\right)\left(\frac{\log n}{n}\right)^{3} \\
& =4 n \frac{\sin (n+1) \theta}{\sin \theta}(1-\cos \theta)^{2}+(1-\cos \theta) O(n \log n)+O(\log n)
\end{aligned}
$$

(3.12) $\psi_{n}^{(1)}(r, \theta)=16 \frac{(\log n)^{3}}{n^{2}}+8 \log n(1-\cos \theta)$

$$
\begin{aligned}
-\frac{\log n}{n^{2}} e^{-p}\left[4 n \frac{\sin (n+1) \theta}{\sin \theta}(1-\cos \theta)^{2}\right. & \\
& +(1-\cos \theta) O(n \log n)+O(\log n)] \\
= & 16 \frac{(\log n)^{3}}{n^{2}}(1+o(1))+8 \log n(1+o(1))(1-\cos \theta) \\
& -4 \frac{\log n}{n} e^{-p \sin (n+1) \theta}(1-\cos \theta)^{2} .
\end{aligned}
$$

Thus the essential part of $\psi_{n}^{(1)}(r, \theta)$ is the expression

$$
4(1-\cos \theta) \log n\left[2-\frac{e^{-p}}{n} \cdot \frac{\sin (n+1) \theta}{\sin \theta}(1-\cos \theta)\right] .
$$

When $n$ is even, the minimum of the square bracket in (3.13) is reached for $\theta=\pi$. Thus $1-e^{-p}$ must be non-negative. If $p$ denotes a bounded 
function of $n, p(n)$, we then have $\lim _{n \rightarrow \infty} p(2 n)=0$.

If $n$ is odd, we let $-\mu=-0.217 \cdots=$ the absolute minimum of $\sin h / h$, which occurs in $\pi<h<\frac{3 \pi}{2}$. If $c_{0}$ is a sufficiently large constant it is easily seen that the square bracket in (3.13) is positive for

$$
0<\frac{c_{0}}{n+1}<\theta<\pi-\frac{c_{0}}{n+1}
$$

and that its minimum occurs in the interval $\pi-\left\{\left(c_{0}\right) / n+1\right\}<\theta<\pi$ for large odd values of $n$. Let $\theta=\pi-\{(h) / n+1\}$. Then for $n$ odd

$$
\begin{aligned}
{\left[2-\frac{e^{-p}}{n} \frac{\sin (n+1) \theta}{\sin \theta}(1-\cos \theta)\right]=2\left(1+e^{-p} \frac{\sin h}{h}\right)+O\left(1 / n^{2}\right) } \\
=2\left(1-\mu e^{-p}\right)+O\left(n^{-2}\right) .
\end{aligned}
$$

It follows that

$$
\lim _{n \rightarrow \infty} p(2 n+1)=\log \mu=-1.527 \cdots .
$$

It follows from the discussion above that we have

$$
R_{n}^{(1)}=1-2 \frac{\log n}{n}+\frac{\log \log n}{n}+\frac{\beta}{n}+o(1 / n)
$$

where $\beta=0=-\log 1$, if $n$ is even, and where

$$
\beta=-\log \left\{\max _{\pi \leqq h \leqq \frac{3 \pi}{2}}\left|\frac{\sin h}{h}\right|\right\}=-\log \mu=1.527 \ldots
$$

when $n$ is odd. This completes the proof of Theorem 1 for the case $k=1$.

We note that for $0 \leqq r \leqq 1 / 2, \psi_{n}^{(1)}(r, \theta) \geqq 0$ for all $n$ and all $\theta$. Indeed, when $r=1 / 2$, we obtain from (3.1) that $\psi_{n}^{(1)}(1 / 2, \theta) \geqq 0$ provided

$$
\begin{aligned}
(30 n & +44) \sin \theta-(12 n+32) \sin 2 \theta-(2 n+4) 2^{-n} \sin (n-1) \theta \\
& +(13 n+24) 2^{-n} \sin n \theta-(30 n+48) 2^{-n} \sin (n+1) \theta \\
& +(28 n+32) 2^{-n} \sin (n+2) \theta-8 n \cdot 2^{-n} \sin (n+3) \theta \\
& \geqq 0,0<\theta<\pi
\end{aligned}
$$

Since $|\sin k \theta / \sin \theta| \leqq k, k=1,2, \cdots,(3.15)$ is satisfied if

$$
(6 n-20) 2^{n} \geqq 73 n^{2}+192 n+108 \text {. }
$$

It is easily verified that (3.17) is true for $n>7$. For $1 \leqq n \leqq 7$ the author has verified that $\psi_{n}(1 / 2, \theta) \geqq 0$. The calculations are simple but somewhat tedious, and will be omitted. 
4. Proof of Theorem 1 for $k=2$. From (2.20) we have

$$
\psi_{n}^{(2)}(r, \theta)=P_{n}(r, \theta)-r^{n} \sum_{j=0}^{5}(-1)^{j} C_{j} \frac{\sin (n-1+j) \theta}{\sin \theta}
$$

where

$$
\begin{aligned}
P_{n}(r, \theta)= & \left\{n(n+1)+\left(2 n^{2}+18 n\right) r^{2}-\left(2 n^{2}-6 n-36\right) r^{4}\right. \\
& -\left\{\left(2 n^{2}+6 n\right) r+(16 n+24) r^{3}-\left(2 n^{2}+6 n\right) r^{5}\right\} \frac{\sin 2 \theta}{\sin \theta} \\
& +\left\{\left(n^{2}+5 n+6\right) r^{2}-\left(n^{2}+n\right) r^{4}\right\} \frac{\sin 3 \theta}{\sin \theta} \\
= & A+B(1-\cos \theta)+C(1-\cos \theta)^{2},
\end{aligned}
$$

(4.3) $A=n^{2}+n-\left(4 n^{2}+12 n\right) r+\left(5 n^{2}+33 n+18\right) r^{2}-(32 n+48) r^{3}$

$$
\begin{aligned}
& -\left(5 n^{2}-3 n-36\right) r^{4}+\left(4 n^{2}+12 n\right) r^{5}-\left(n^{2}+5 n+6\right) r^{6} \\
= & -(1-r)^{6}\left(n^{2}+5 n+6\right)+(1-r)^{5}\left(2 n^{2}+18 n+36\right) \\
& -(1-r)^{4}(12 n+54)+24(1-r)^{3},
\end{aligned}
$$

(4.4) $B=\left(4 n^{2}+12 n\right) r-\left(8 n^{2}+40 n+48\right) r^{2}+(32 n+48) r^{3}$

$$
\begin{aligned}
& +\left(8 n^{2}+8 n\right) r^{4}-\left(4 n^{2}+12 n\right) r^{5} \\
= & -r(1-r)^{1}\left(4 n^{2}+12 n\right)+r(1-r)^{3}\left(8 n^{2}+40 n\right) \\
& -r(1-r)^{2}(16 n-48)-48 r(1-r) .
\end{aligned}
$$

(4.5) $C=\left(4 n^{2}+20 n+24\right) r^{2}-\left(4 n^{2}+4 n\right) r^{4}$

$C_{0}=(2 n+6) r^{6}$

$C_{1}=(8 n+24) r^{5}+2 n r^{7}$

$C_{2}=(12 n+36) r^{4}+8 n r^{6}$

(4.6) $\quad C_{3}=(8 n+24) r^{3}+12 n r^{5}$

$C_{4}=(2 n+6) r^{2}+8 n r^{4}$

$C^{5}=$

$2 n r^{3}$.

Letting

$$
\begin{gathered}
r=e^{-\varepsilon}, \quad \varepsilon=\frac{\log n}{n}-\frac{\log \log n}{n}+\frac{q}{n}, \\
r^{n}=\frac{\log n}{n} e^{-q}, \quad 1-r=1-\frac{\log n}{n}+\frac{\log \log n-q}{n}+O\left(\left(\frac{\log n}{n}\right)^{2}\right),
\end{gathered}
$$

we obtain

$$
A \cong 2 \frac{(\log n)^{5}}{n^{3}}, \quad B \cong 8 \frac{(\log n)^{3}}{n}, \quad C \cong 8 n \log n,
$$




$$
\begin{aligned}
& C_{0}=(2 n+6)-(12 n+36) \varepsilon+(36 n+108) \varepsilon^{2} \\
&-(72 n+216) \varepsilon^{3}+108 n \varepsilon^{4}+O\left(n \varepsilon^{5}\right) \\
& C_{1}=(10 n+24)-(54 n+120) \varepsilon+(149 n+300) \varepsilon^{2} \\
&-(281 n+500) \varepsilon^{3}+\frac{4901}{12} n \varepsilon^{4}+O\left(n \varepsilon^{5}\right) \\
& C_{2}=(20 n+36)-(96 n+144) \varepsilon+(240 n+288) \varepsilon^{2} \\
&-(416 n+384) \varepsilon^{3}+560 n \varepsilon^{4}+O\left(n \varepsilon^{5}\right) \\
& C_{3}=(20 n+24)-(84 n+72) \varepsilon+(186 n+108) \varepsilon^{2} \\
&-(286 n+108) \varepsilon^{3}+\frac{679}{2} n \varepsilon^{4}+O\left(n \varepsilon^{5}\right) \\
& C_{4}=(10 n+6)-(36 n+12) \varepsilon+(68 n+12) \varepsilon^{2} \\
&-(88 n+8) \varepsilon^{3}+\frac{260}{3} n \varepsilon^{4}+O\left(n \varepsilon^{5}\right) \\
& C_{5}=2 n-6 n \cdot \varepsilon+9 n \cdot \varepsilon^{2}-9 n \cdot \varepsilon^{3}+\frac{27}{4} n \varepsilon^{4}+O\left(n \varepsilon^{5}\right) .
\end{aligned}
$$

We now write

(4.9) $\psi_{n}^{(2)}(r, \theta)=A+B(1-\cos \theta)+C(1-\cos \theta)^{2}-r^{n} \cdot \sum_{j=0}^{5}(-1)^{\jmath} D_{j} \varepsilon^{\jmath}$.

From (4.1), (4.2), and (4.8), we find

$$
\begin{aligned}
& \begin{array}{c}
D_{0} \cdot \sin \theta=(2 n+6) \sin (n-1) \theta-(10 n+24) \sin n \theta \\
-(20 n+36) \sin (n+1) \theta+(20 n+24) \sin (n+2) \theta \\
-(10 n+6) \sin (n+3) \theta+2 n \sin (n+4) \theta, \\
D_{0}=\left[\begin{array}{r}
\cos (2 n+3) \frac{\theta}{2} \\
-8 n \cdot \frac{\sin (n+1) \theta}{\sin \theta}
\end{array}\right](1-\cos \theta)^{2} . \\
D_{1} \cdot \sin \theta=(12 n+36) \sin (n-1) \theta-(54 n+120) \sin n \theta \\
+(96 n+144) \sin (n+1) \theta-(84 n+72) \sin (n+2) \theta \\
\quad+(36 n+12) \sin (n+3) \theta-6 n \sin (n+4) \theta .
\end{array}
\end{aligned}
$$

By Lemma 1, we obtain

$$
\begin{aligned}
& D_{1}=\left[n(1-\cos \theta)^{2}+(1-\cos \theta] \cdot[O(n)+O(1)] \cdot O(1)\right. \\
& =\left[n^{2}(1-\cos \theta)^{2}+n(1-\cos \theta)\right] \cdot O(1) . \\
& \begin{aligned}
D_{2} \cdot \sin \theta= & (36 n+108) \sin (n-1) \theta-(149 n+300) \sin n \theta \\
& \quad+(240 n+288) \sin (n+1) \theta-(186 n+108) \sin (n+2) \theta \\
& \quad+(68 n+12) \sin (n+3) \theta-9 n \sin (n+4) \theta
\end{aligned}
\end{aligned}
$$




$$
\begin{aligned}
D_{2}=\left[n^{2}(1-\cos \theta)^{2}+n(1-\cos \theta)\right] & \cdot O(1) \\
+ & {[n(1-\cos \theta)+(1-\cos \theta)] \cdot O(1) . }
\end{aligned}
$$

$D_{3} \cdot \sin \theta=(72 n+216) \sin (n-1) \theta-(281 n+500) \sin n \theta$

$$
+(416 n+384) \sin (n+1) \theta-(286 n+108) \sin (n+2) \theta
$$$$
+(88 n+8) \sin (n+3) \theta-9 n \sin (n+4) \theta \text {, }
$$$$
D_{3}=\{n(1-\cos \theta)+1\} \cdot n \cdot O(1)=\left[n^{2}(1-\cos \theta)+n\right] O(1) \text {. }
$$$$
D_{4} \cdot \sin \theta=108 n \sin (n-1) \theta-\frac{4901}{12} n \sin n \theta+560 n \sin (n+1) \theta
$$$$
-\frac{679}{2} \sin (n+2) \theta+\frac{260}{3} n \sin (n+3) \theta-\frac{27}{4} n \sin (n+4) \theta,
$$

$$
D_{4}=\{n(1-\cos \theta)+1\} \cdot n \cdot O(1)=\left[n^{2}(1-\cos \theta)+n\right] \cdot O(1) .
$$

$$
D_{5}=O(n) \cdot \sum_{j=0}^{5}\left|\frac{\sin (n-1+j) \theta}{\sin \theta}\right|=O\left(n^{2}\right) \text {. }
$$

$$
\begin{aligned}
& \quad D_{0}-D_{1} \varepsilon+D_{2} \varepsilon^{2}-D_{3} \varepsilon^{3}+D_{4} \varepsilon^{4}-D_{5} \varepsilon^{5} \\
& =\left[-8 n \frac{\cos (2 n+3) \theta / 2}{\cos \theta / 2}+24 \frac{\sin (n+1) \theta]}{\sin \theta}\right](1-\cos \theta)^{2} \\
& +\left[n^{2}(1-\cos \theta)^{2}+n(1-\cos \theta)\right] O(1) \frac{\log n}{n} \\
& +\left[n^{2}(1-\cos \theta)^{2}+n(1-\cos \theta)\right] O(1)\left(\frac{\log n}{n}\right)^{2} \\
& +\left[n^{2}(1-\cos \theta)+n\right] O(1)\left(\frac{\log n}{n}\right)^{3} \\
& +\left[n^{2}(1-\cos \theta)+n\right] O(1)\left(\frac{\log n}{n}\right)^{4}+O\left(n^{2}\right)\left(\frac{\log n}{n}\right)^{5} \\
& =\left[-8 n \frac{\cos (2 n+3) \theta / 2}{\cos \theta / 2}+24 \frac{\sin (n+1) \theta}{\sin \theta}+O(n \log n)\right](1-\cos \theta)^{2} \\
& +(1-\cos \theta) \cdot O(\log n)+O\left(\frac{(\log n)^{3}}{n^{2}}\right) .
\end{aligned}
$$

From (4.7), (4.9), and (4.10), we have

$$
\begin{aligned}
\psi_{n}^{(2)}(r, \theta)= & \frac{2(\log n)^{5}}{n^{3}}+\frac{8(\log n)^{3}}{n}(1-\cos \theta)+8 n \log n(1-\cos \theta)^{2} \\
& -\frac{\log n}{n} e^{-q}\left[-\frac{\cos (2 n+3) \theta / 2}{\cos \theta / 2}+24 \frac{\sin (n+1) \theta}{\sin \theta}\right. \\
& +O(n \log n)](1-\cos \theta)^{2} \\
& -\frac{\log n}{n} e^{-q}\left[(1-\cos \theta) O(\log n)+O\left(\frac{(\log n)^{3}}{n^{2}}\right)\right]
\end{aligned}
$$




$$
\begin{aligned}
= & {\left[2 \frac{(\log n)^{5}}{n^{3}}-e^{-q} \cdot O\left(\frac{(\log n)^{4}}{n^{3}}\right)\right] } \\
& +\left[8 \frac{(\log n)^{3}}{n}-e^{-q} \cdot O\left(\frac{(\log n)^{2}}{n}\right)\right](1-\cos \theta) \\
& +8 \log n\left[n+e^{-q} \frac{\cos (2 n+3) \theta / 2}{\cos \theta / 2}-\frac{24 e^{-q}}{n} \frac{\sin (n+1) \theta}{\sin \theta}\right. \\
& \left.+e^{-q} \cdot O(\log n)\right](1-\cos \theta)^{2} .
\end{aligned}
$$

From (4.11) it is seen that we must have the quantity $L \geqq 0$ where

$$
L=1+e^{-q}\left[\frac{\cos (2 n+3) \theta / 2}{n \cos (\theta / 2)}-\frac{24}{n^{2}} \frac{\sin (n+1) \theta}{\sin \theta}\right] .
$$

For $n$ even the minimum of $L$ is attained for $\theta=\pi$ and equals

$$
1-e^{-q}\left(\frac{2 n+3}{n}+\frac{24(n+1)}{n^{2}}\right)=1-2 e^{-q}+e^{-q} \cdot o\left(\frac{1}{n}\right) .
$$

Thus if $q=q(n)$, a bounded function of $n$, we require

$$
\lim _{n \rightarrow \infty} q(2 n)=\log 2 \text {. }
$$

If $n$ is odd, we let $\theta=\pi-(2 h) / 2 n+3$ and find that

$$
L \cong 1+2 e^{-q} \frac{\sin h}{h}
$$

and

$$
\min _{\theta} L \cong 1-2 \mu e^{-q}
$$

where

$$
\mu=0.217 \cdots=\max _{\pi \leqq h \leqq \frac{3 \pi}{2}}\left|\frac{\sin h}{h}\right|, \quad \text { and } \quad \lim _{n \rightarrow \infty} q(2 n-1)=\log (2 \mu) .
$$

It follows that we have

$$
R_{n}^{(2)}=1-\frac{\log n}{n}+\frac{\log \log n}{n}-\frac{\gamma}{n}+o\left(\frac{1}{n}\right)
$$

where

$$
\gamma= \begin{cases}\log 2, & \text { if } n \text { is even } \\ \log (2 \mu), & \text { if } n \text { is odd }\end{cases}
$$

This completes the proof of Theorem 1 for the case $k=2$.

In the case $k=0$, which was investigated by Szász [8], if we employ procedures analogous to those above for $k=1$ and 2 , we are led to the expression 


$$
3 \frac{\log n}{n}-\frac{\log n}{n^{3}} e^{-t} \cdot 2 n(1-\cos \theta) \frac{\cos (2 n+1) \theta / 2}{\cos \theta / 2}
$$

when

$$
r=1-3 \frac{\log n}{n}+\frac{\log \log n}{n}-\frac{t}{n} .
$$

With arguments similar to those used above, we find that the "correct" value of $t$ is $\log (4 / 3)$ when $n$ is even (as Szász obtained [8]), and $\log (4 \mu / 3)$ when $n$ is odd, the latter result being new.

5. Proof of Theorem 1 for $k=3$. The theorem of Fejér [2], quoted in the introduction, states that

$$
R_{n}^{(3)} \geqq 1,
$$$$
n=1,2,3, \cdots \text {. }
$$

We shall give a new and simple proof of (5.1), and also give a demonstration of the sharpened result

$$
R_{2 n}^{(3)}=1, \quad R_{2 n-1}^{(3)}>1, \quad n=1,2,3, \cdots,
$$

and

$$
\limsup _{n \rightarrow \infty}(2 n-1)\left(R_{2 n-1}^{(3)}-1\right) \leqq \alpha_{0}=1.07 \cdots
$$

where $\alpha_{0}$ is the positive root of the equation

$$
3-\alpha-3 \mu e^{\alpha}=0
$$

where

$$
\mu=\max _{\pi \leqq h \leqq \frac{3 \pi}{2}}\left|\frac{\sin h}{h}\right|=0.217 \cdots .
$$

From (2.7) we have

$$
\begin{aligned}
6(1-z)^{5} S_{n-2}^{(3)}(z)= & n(n-1)(n-2) z-3(n-1)\left(n^{2}-4\right) z^{2} \\
& +3(n+1)\left(n^{2}-4\right) z^{3}-n(n+1)(n+2) z^{4} \\
& +6(n+2) z^{n+2}-6(n-2) z^{n+3} .
\end{aligned}
$$

Letting $z=e^{i \theta}$ in (5.5) we have for $n>2$

$$
\begin{aligned}
\Im S_{n-2}^{(3)}(z)= & \frac{1}{32 \sin ^{5}(\theta / 2)}\left[\left(n^{2}-4\right) \cos \frac{\theta}{2}-n^{2} \cos \frac{3 \theta}{2}\right. \\
& \left.\quad+(n+2) \cos (2 n-1) \frac{\theta}{2}-(n-2) \cos (2 n+1) \frac{\theta}{2}\right] \\
= & \frac{\sin \theta}{16 \sin ^{4}(\theta / 2)}\left[n^{2}+n \frac{\sin n \theta}{\sin \theta}-2\left(\frac{\sin n \theta / 2}{\sin \theta / 2}\right)^{2}\right] .
\end{aligned}
$$


In earlier papers we have shown [5], [6], that

$$
n^{2}+n \frac{\sin n \theta}{\sin \theta}-2\left(\frac{\sin n \theta / 2}{\sin \theta / 2}\right)^{2} \geqq 0,
$$

for all integers $n$ and all $\theta$. From (5.6) and (5.7) we have at once that

$$
\Im S_{n}^{(3)}\left(e^{i \theta}\right) \geqq 0, \quad 0 \leqq \theta \leqq \pi, \quad n=1,2, \cdots .
$$

However, the function

$$
F(z)=\left(z^{-1}-z\right) S_{n}^{(3)}(z)
$$

is analytic in $|z| \leqq 1$, and $\Re F\left(e^{i \theta}\right)=2 \sin \theta \Im S_{n}^{(3)}\left(e^{i \theta}\right) \geqq 0$. Since the minimum of the harmonic function $\Re F(z)$ in $|z| \leqq 1$ occurs on $|z|=1$ we have $\Re F(z)>0$ for $|z|<1$. From the representation (5.9) it follows from the work of Rogosinski [7] that $S_{n}^{(3)}(z)$ is typically-real in the unit circle, which is to say that

$$
\Im S_{n}^{(3)}\left(r e^{i \theta}\right) \geqq 0, \quad 0 \leqq \theta \leqq \pi, 0 \leqq r \leqq 1 .
$$

The theorem of Fejér, or inequality (5.1) follows from (5.10) and the remarks made in section two.

We now attack the problem from an alternative point of view for the case $k=3$. From (2.17) and (5.6) we write

$$
\begin{aligned}
\psi_{n}^{(3)}(r, \theta)= & 384 \sin ^{6} \frac{\theta}{2}\left[(n+2)^{2}+(n+2) \frac{\sin (n+2) \theta}{\sin \theta}\right. \\
& +\left[\psi_{n}^{(3)}(r, \theta)-\psi_{n}^{(3)}(1, \theta)\right] .
\end{aligned}
$$

Let $\quad r=1+\alpha / n$ where $\alpha=\alpha(n)=O(1)>0$. Then $r^{k}-1=$ $k \alpha / n+O\left(\alpha^{2} / n^{2}\right), \quad k=$ positive integer independent of $n, r^{n+k}-1=$ $e^{\alpha}-1+n^{-1} k \alpha e^{\alpha}+O\left(n^{-1} \alpha^{2} e^{\alpha}\right)$. From (2.21) and (5.11) we then obtain for $r=1+\alpha / n$ asymptotically,

$$
\begin{aligned}
\psi_{n}^{(3)}(r, \theta)-\psi_{n}^{(3)}(1, \theta) \cong 28 n^{2} \alpha & +56 n^{2} \alpha \cos \theta-12 n^{2} \alpha\left(4 \cos ^{2} \theta-1\right) \\
& \quad-2 n^{2} \alpha\left(4 \cos \theta-8 \cos ^{3} \theta\right)-\frac{\left(e^{\alpha}-1\right) S}{\sin \theta} \\
= & -128 n^{2} \alpha \sin ^{6} \frac{\theta}{2}-\frac{\left(e^{\alpha}-1\right) S}{\sin \theta},
\end{aligned}
$$

where

$$
\begin{aligned}
S= & 6 n \sin (n-1) \theta-36 n \sin n \theta+90 n \sin (n+1) \theta-120 n \sin (n+2) \theta \\
& +90 n \sin (n+3) \theta-36 n \sin (n+4) \theta+6 n \sin (n+5) \theta \\
= & -384 n \sin (n+2) \theta \sin ^{6} \frac{\theta}{2},
\end{aligned}
$$




$$
\psi_{n}^{(3)}(r, \theta)-\psi_{n}^{(3)}(1, \theta) \cong 128 \sin ^{6} \frac{\theta}{2}\left[3 n\left(e^{\alpha}-1\right) \frac{\sin (n+2) \theta}{\sin \theta}-\alpha n^{2}\right] .
$$

Since for sufficiently large values of $n$ we can only have

$$
\psi_{n}^{(3)}(r, \theta) \geqq 0 \quad \text { or } \quad r=1+n^{-1} \alpha, \alpha=\alpha(n)>0,
$$

provided

$$
\begin{aligned}
& 3\left[(n+2)^{2}+(n+2) \frac{\sin (n+2) \theta}{\sin \theta}-2\left(\frac{\sin (n+2) \theta / 2}{\sin \theta / 2}\right)^{2}\right] \\
& +3\left(e^{\alpha}-1\right) n \frac{\sin (n+2) \theta}{\sin \theta}-n^{2} \alpha \geqq 0,
\end{aligned}
$$

we see that, when $n$ is even and $\theta=\pi$, we must have

$$
-3\left(e^{\alpha}-1\right) n(n+2)-n^{2} \alpha \geqq 0 .
$$

(5.15) implies that $\alpha$ is non-positive, contrary to our assumption that $\alpha>0$. Hence $\alpha=0$ for $n$ even and sufficiently large. However, it is easily seen that $\alpha=0$ for all even $n$ by the following argument. Since

$$
S_{n}^{(3)}(z)=\sum_{\nu=1}^{n} \nu C_{3}^{n+3-\nu} z^{\nu},
$$

and because of the identity

$$
\sum_{\nu=1}^{n}(-1)^{\nu} \nu^{2}(n+1-\nu)(n+2-\nu)(n+3-\nu)=0, \quad n \text { even, }
$$

it follows that the derivative of $S_{n}^{(3)}(z)$ vanishes at $z=-1$ for $n$ even. $S_{n}^{(3)}(z)$, typically-real in $|z| \leqq 1$, therefore cannot be typically-real in $|z|$ $\leqq r$ for $r>1, n$ even. Thus $\alpha=0$ for all even $n$, and $R_{2 n}^{(3)}=1, n=$ $1,2, \cdots$.

The situation for $n$ odd is not so simple. Fejér has pointed out [2] that $\Im S_{n}^{(3)}\left(e^{i \theta}\right)>0,0<\theta<\pi$, from which it follows that $\psi_{n}^{(3)}(1, \theta)>0$ for all $\theta$ with the possible exception of the values $\theta=0$ and $\pi$. When $n$ is odd, however, it is easily seen from (5.6) and (2.17) that $\psi_{n}^{(3)}(1, \pi)>0$. From (5.16) it also follows that

$$
\lim _{\theta \rightarrow 0} \frac{\Im S_{n}^{(3)}\left(e^{i \theta}\right)}{\sin \theta}=\sum_{v=1}^{n} \nu^{2} C_{3}^{(n+3-\nu)}>0 .
$$

Consequently $\Im S_{n}^{(3)}\left(e^{i \theta}\right) \cdot \operatorname{cosec} \theta>0$ for all $\theta$ when $n$ is odd, and so $R_{2 n-1}^{(3)}>1, n=1,2, \cdots$. To obtain an asymptotic upper bound for $R_{2 n-1}^{(3)}$ we shall show that (5.14) is not verified, when $n$ is sufficiently large, for all $\theta$ when $\alpha$ exceeds $\alpha_{0}=1.07 \cdots, \alpha_{0}$ being the positive root of the equation (5.4).

Letting $\theta=\pi-[h /(n+2)], n$ odd, we find that the left hand side of inequality (5.14) is asymptotically equal to the expression 


$$
3 n^{2}\left[1+\frac{\sin h}{h}-O\left(n^{-2}\right)\right]+3 n^{2}\left(e^{\alpha}-1\right)\left(\frac{\sin h}{h}+O\left(n^{-1}\right)\right)-n^{2} \alpha,
$$

from which (5.4) and (5.3) follow. It should be noticed that the constant $\alpha_{0}$ in (5.3) could be replaced by a smaller one. Indeed, for $\theta=x / n$, the left-hand side of (5.14) is asymptotically equal to

$$
n^{2}\left[3-\alpha+3 e^{\alpha} \frac{\sin x}{x}-6\left(\frac{\sin x / 2}{x / 2}\right)^{2}\right] .
$$

Calculation of the smallest positive $\alpha$ for which the expression (5.18) is non-positive for some $x \geqq \pi$ would lead to a smaller constant to replace $\alpha_{0}$.

From (2.3) and (5.1) it follows at once that $R_{n}^{(k)} \geqq 1$ for $k \geqq 3$ and all positive integers $n$.

\section{REFERENCES}

1. R. K. Brown, On the partial sums of a typically-real function, Master of Science thesis (1950), Rutgers University Library.

2. L. Fejér, Neue Eigenschaften der Mittelwerte bei den Fourierreihen, J. London Math. Soc. 8 (1933), 53-62.

3. M. S. Robertson, On the coefficients of a typically-real function, Bull. Amer. Math. Soc. 41 (1935), 565-572.

4. _ Applications of a lemma of Fejér to typically-real functions, Proc. Amer. Math. Soc. 1 (1950), 555-561.

5 . _ - Univalenl power series with multiply monotonic sequences of coefficients, Ann. of Math. 46 (1945), 533-55.

6. ____, The coefficients of univalent functions, Bull. Amer. Math. Soc. 51 (1945), 733-738.

7. W. Rogosinski, Über positive harmonische Entwicklungen und typischreelle Potenzreihen, Math. Z. 35 (1932), 93-121.

8. O. Szász, On the partial sums of harmonic developments and of power series, Trans. Amer. Math. Soc. 52 (1942), 12-21.

9. P. Turán, Über die monotone Konvergenz der Cesàro-mittel bei Fourier-und Potenzreihen, Proc. Cambridge Philos. Soc. 34 (1938), 134-143.

Rutgers-The State University of New JerSey. 


\section{PACIFIC JOURNAL OF MATHEMATICS}

\section{EDITORS}

\section{David Gilbarg}

Stanford University

Stanford, California

\section{R. A. Beaumont}

University of Washington

Seattle 5, Washington

\author{
A. L. Whiteman
}

University of Southern California Los Angeles 7, California

E. G. Straus

University of California

Los Angeles 24, California

\section{ASSOCIATE EDITORS}

\author{
E. F. BECKENBACH \\ C. E. BURGESS \\ M. HALL \\ E. HEWITT
}
A. HORN
V. GANAPATHY IYER
R. D. JAMES
M. S. KNEBELMAN
L. NACHBIN
I. NIVEN
T. G. OSTROM
H. L. ROYDEN

M. M. SCHIFFER

G. SZEKERES

F. WOLF

K. YOSIDA

\section{SUPPORTING INSTITUTIONS}

\author{
UNIVERSITY OF BRITISH COLUMBIA \\ CALIFORNIA INSTITUTE OF TECHNOLOGY \\ UNIVERSITY OF CALIFORNIA \\ MONTANA STATE UNIVERSITY \\ UNIVERSITY OF NEVADA \\ OREGON STATE COLLEGE \\ UNIVERSITY OF OREGON \\ OSAKA UNIVERSITY \\ UNIVERSITY OF SOUTHERN CALIFORNIA
}

\author{
STANFORD UNIVERSITY \\ UNIVERSITY OF TOKYO \\ UNIVERSITY OF UTAH \\ WASHINGTON STATE COLLEGE \\ UNIVERSITY OF WASHINGTON \\ * * * * \\ AMERICAN MATHEMATICAL SOCIETY \\ CALIFORNIA RESEARCH CORPORATION \\ HUGHES AIRCRAFT COMPANY \\ THE RAMO-WOOLDRIDGE CORPORATION
}

Mathematical papers intended for publication in the Pacific Journal of Mathematics should be typewritten (double spaced), and the author should keep a complete copy. Manuscripts may be sent to any one of the four editors. All other communications to the editors should be addressed to the managing editor, E. G. Straus at the University of California, Los Angeles 24, California.

50 reprints per author of each article are furnished free of charge; additional copies may be obtained at cost in multiples of 50 .

The Pacific Journal of Mathematics is published quarterly, in March, June, September, and December. The price per volume (4 numbers) is $\$ 12.00$; single issues, $\$ 3.50$. Back numbers are available. Special price to individual faculty members of supporting institutions and to individual members of the American Mathematical Society: $\$ 4.00$ per volume; single issues, $\$ 1.25$.

Subscriptions, orders for back numbers, and changes of address should be sent to Pacific Journal of Mathematics, 2120 Oxford Street, Berkeley 4, California.

Printed at Kokusai Bunken Insatsusha (International Academic Printing Co., Ltd.), No. 6, 2-chome, Fujimi-cho, Chiyoda-ku, Tokyo, Japan.

PUBLISHED BY PACIFIC JOURNAL OF MATHEMATICS, A NON-PROFIT CORPORATION

The Supporting Institutions listed above contribute to the cost of publication of this Journal, but they are not owners or publishers and have no responsibility for its content or policies. 


\section{Pacific Journal of Mathematics}

\section{Vol. 8, No. $4 \quad$ June, 1958}

Richard Arens, The maximal ideals of certain functions algebras ........ 641

Glen Earl Baxter, An operator identity ........................... 649

Robert James Blattner, Automorphic group representations ........... 665

Steve Jerome Bryant, Isomorphism order for Abelian groups ............ 679

Charles W. Curtis, Modules whose annihilators are direct summands...... 685

Wilbur Eugene Deskins, On the radical of a group algebra ............ 693

Jacob Feldman, Equivalence and perpendicularity of Gaussian

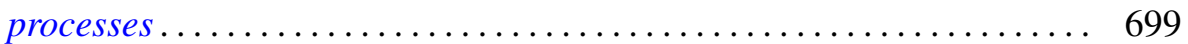

Marion K. Fort, Jr. and G. A. Hedlund, Minimal coverings of pairs by triples....................................... 709

I. S. Gál, On the theory of $(m, n)$-compact topological spaces ......... 721

David Gale and Oliver Gross, A note on polynomial and separable games........................................ 735

Frank Harary, On the number of bi-colored graphs ............... 743

Bruno Harris, Centralizers in Jordan algebras ................... 757

Martin Jurchescu, Modulus of a boundary component ............... 791

Hewitt Kenyon and A. P. Morse, Runs . . . . . . . . . . . . . . . . . . . . . . 811

Burnett C. Meyer and H. D. Sprinkle, Two nonseparable complete metric

spaces defined on $[0,1] \ldots \ldots \ldots \ldots \ldots \ldots \ldots \ldots \ldots \ldots \ldots . \ldots . \ldots . \ldots . \ldots 25$

M. S. Robertson, Cesàro partial sums of harmonic series expansions...... 829

John L. Selfridge and Ernst Gabor Straus, On the determination of numbers by their sums of a fixed order ........................ 847

Annette Sinclair, A general solution for a class of approximation

problems .................................

George Szekeres and Amnon Jakimovski, $(C, \infty)$ and $(H, \infty)$ methods of summation...................................... 867

Hale Trotter, Approximation of semi-groups of operators. ............. 887

L. E. Ward, A fixed point theorem for multi-valued functions ........... 921

Roy Edwin Wild, On the number of lattice points in $x^{t}+y^{t}=n^{t / 2} \ldots \ldots .929$ 